\title{
A Rare Case of Primary Aortoduodenal Fistula
}

\author{
Hyun Jin Bae, Byung-Wook Kim, Joon Sung Kim
}

Division of Gastroenterology, Department of Internal Medicine, Incheon St. Mary's Hospital, College of Medicine, The Catholic University of Korea, Incheon, Korea

\begin{abstract}
Aortoduodenal fistula is a rare but life-threatening condition that can cause gastrointestinal bleeding. Due to its rarity, it is often overlooked as a cause of gastrointestinal blood loss. Notably, the mortality rate of aortoduodenal fistula is nearly $100 \%$ in undiagnosed and untreated cases. We report a case of aortoduodenal fistula, which resulted in the patient's death. This report highlights the importance of considering even extremely rare causes of gastrointestinal bleeding in the differential diagnosis in patients with such a presentation. (Korean J Helicobacter Up Gastrointest Res 2020;20:164-166)
\end{abstract}

Key Words: Aortic aneurysm, abdominal; Endoscopy; Fistula

\section{INTRODUCTION}

Aortoduodenal fistula is an abnormal communication between the aorta and the duodenum. ${ }^{1-3}$ It is a rare but fatal cause of gastrointestinal bleeding. ${ }^{2,3}$ Early diagnosis is challenging, since there are not always active bleeding on the endoscopy. ${ }^{2,45}$ Thus, abdominal CT with intravenous contrast helps a high index of suspicion. ${ }^{4,5}$ Herein, we report a case of aortoduodenal fistula which gives us a lesson that a high index of suspicion even for extremely rare causes is always needed in evaluation of gastrointestinal bleeding. This case is unique in that the fistula formed as a result of an atherosclerotic infrarenal aorta and a visible vessel in the duodenum the etiology of which was not clear.

\section{CASE REPORT}

A 79-year-old man presented to Incheon St. Mary's Hospital because of hematochezia for 3 days. He had no abdominal symptoms, and physical examination was unremarkable. His vital sign was blood pressure of 110/60 $\mathrm{mmHg}$, heart rate of $97 / \mathrm{min}$, respiratory rate of $20 / \mathrm{min}$, and body temperature of $37.1^{\circ} \mathrm{C}$. Laboratory findings were

Received: October 15, 2019 Revised: November 12, 2019 Accepted: November 13, 2019 Corresponding author: Joon Sung Kim

Division of Gastroenterology, Department of Internal Medicine, Incheon St. Mary's Hospital, College of Medicine, The Catholic University of Korea, 56 Dongsu-ro, Bupyeong-gu, Incheon 21431, Korea

Tel: +82-32-280-5052, Fax: +82-32-280-5987, E-mail: kijoons@catholic.ac.kr non-specific except hemoglobin level of $9.9 \mathrm{~g} / \mathrm{dL}$. He had a history of coronary artery disease and received percutaneous coronary intervention 4 years ago. He was taking aspirin due to the past history. Upper gastrointestinal endoscopy showed a visible vessel in the second portion of the duodenum (Fig. 1A). Hemoclipping was performed at the lesion (Fig. 1B) and the patient was admitted to intensive care unit for monitoring. However, after a few hours later from endoscopic hemostasis, the patient's vital sign became unstable. His blood pressure was dropped to $70 / 40 \mathrm{mmHg}$, and heart rate was raised to $160 / \mathrm{min}$. The follow-up hemoglobin level dropped to $5.7 \mathrm{~g} / \mathrm{dL}$. Contrast-enhanced abdominal CT revealed a $4-\mathrm{cm}$ partially thrombosed aneurysm (Fig. 1C) with internal air density in the infrarenal aorta. There was also extravasation of contrast dye into the duodenum suggesting an aortoduodenal fistula (Fig. 1D). The patient planned to receive emergency operation or embolization, however, the patient expired within 12 hours after arriving to Incheon St. Mary's Hospital. As early diagnosis is mandatory for improved survivial, aortoduodenal fistula should be suspected when a visible vessel is present without an ulcer.

\section{DISCUSSION}

Primary aortoenteric fistula is defined as a tract between naïve aorta and any site of gastrointestinal tract, in the absence of aortic reconstructive surgery or other pro-

Copyright $\odot 2020$ Korean College of Helicobacter and Upper Gastrointestinal Research

(a) The Korean Journal of Helicobacter and Upper Gastrointestinal Research is an Open-Access Journal. All articles are distributed under the terms of the Creative Commons Attribution Non-Commercial License (http:// creativecommons.org/licenses/by-nc/4.0) which permits unrestricted non-commercial use, distribution, and reproduction in any medium, provided the original work is properly cited. 

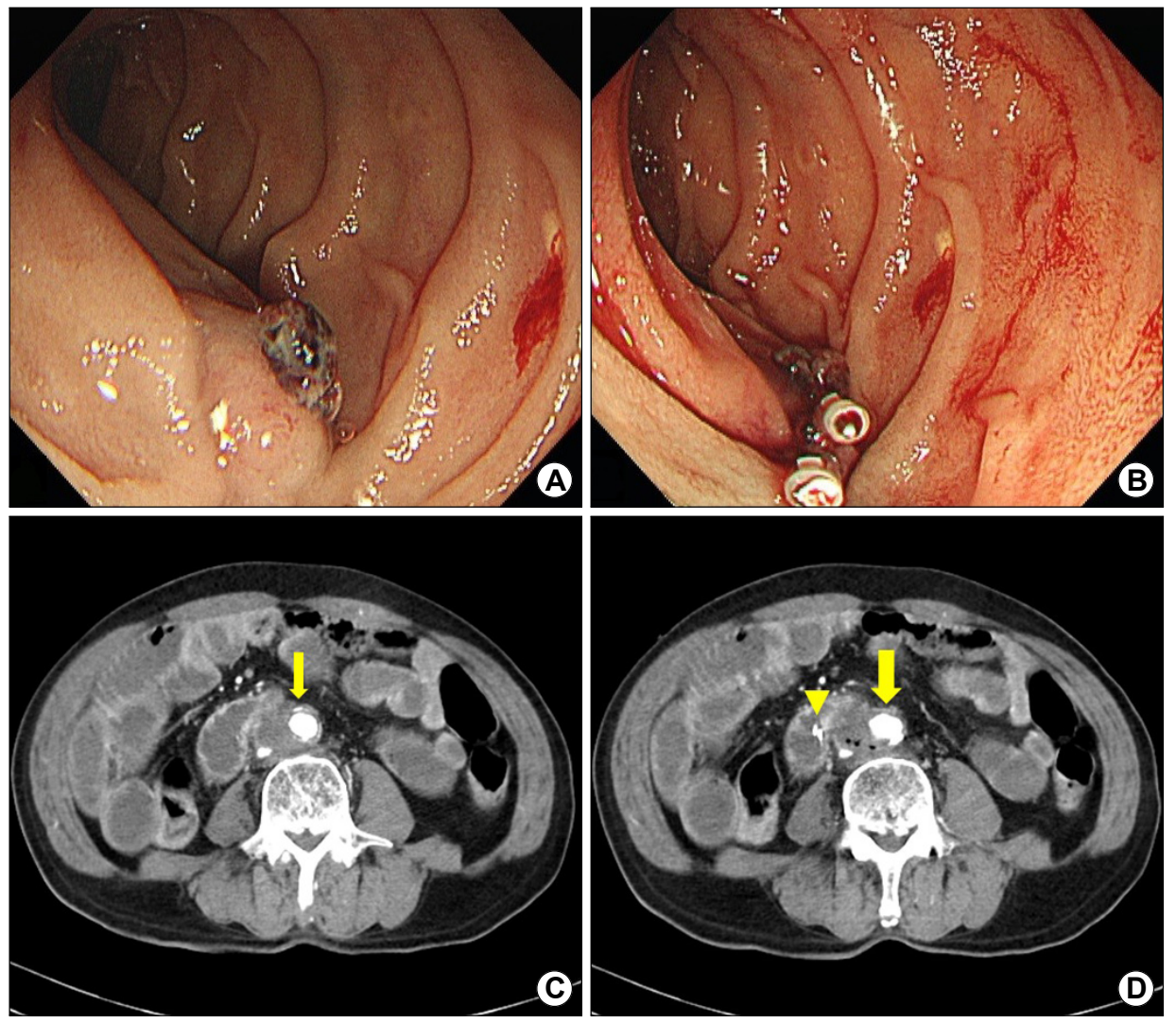

Fig. 1. (A) Endoscopic image showing a vessel in the second portion of the duodenum. (B) Endoscopic image showing injection of epinephrine and clipping of the vessel with two hemoclips for hemostasis. (C) Contrast-enhanced CT scan showing a partially thrombosed aneurysm $(4 \mathrm{~cm})$ of the infrarenal aorta (arrow). (D) Contrast-enhanced CT scan showing extravasation of contrast material (arrowhead) from the aortic aneurysm into the duodenal lumen, visible as an area of internal air density (arrow).

cedures with vascular prostheses. ${ }^{1,2,5,6}$ It is one of extremely rare causes of gastrointestinal bleeding, with an incidence of $0.04 \sim 0.07 \%{ }^{7-10}$ At large, the duodenum is the most common part of aortoenteric fistula, especially the third portion due to its fixed retroperitoneal position with covering the aorta. ${ }^{5-9}$

In 1843 , the first case of primary aortoduodenal fistula was reported. ${ }^{7}$ Since then, about 300 cases have been reported in literature. ${ }^{9,10}$ However, the pathogenesis of primary aortoduodenal fistula is unclear. ${ }^{6}$ The most common cause of primary aortoduodenal fistula is reported as abdominal aortic aneurysm with atherosclerosis. ${ }^{1,25-8}$ Other causes include infection, tumor, foreign body, and radiotherapy. ${ }^{1,4,7,8}$ Clinical triad is known as gastrointestinal bleeding, abdominal pain, and a pulsating abdominal mass, though it occurs in 11 25\% of primary aortoduodenal fistula cases. ${ }^{1-3,8,9}$

Endoscopy is the most common diagnostic tool of choice for upper gastrointestinal bleeding. ${ }^{2-4}$ Unfortunately, endoscopy seldom provides a confirmative diagnostic clue of pri- mary aortoduodenal fistula. ${ }^{1,3,8,9}$ Therefore, a high index of suspicion and an aggressive diagnostic approach is recommended. ${ }^{4,5,8-10}$ If endoscopy is inconclusive for consistent gastrointestinal bleeding, then $\mathrm{CT}$ with contrast should be considered as a next step. ${ }^{1,24}$ Evidences of primary aortoduodenal fistula are an extravasation of contrast from the aorta to the duodenum, a calcified aneurysm with gas in it, an aneurysm with adjacent bowel loop and extraluminal gas in periaortic area, or bowel wall thickening overlying an aneurysm. ${ }^{2,3}$ And if there is no specific focus even in CT, angiography could be an alternative choice.,

The early diagnosis of primary aortoduodenal fistula is extremely difficult because of its nonspecificity and diversity of clinical presentation. ${ }^{1,4,8}$ Not only the diagnosis, but also the treatment is quite challenging. If undiagnosed and untreated, the mortality rate becomes nearly $100 \%{ }^{1,7,9}$ Therefore, if the diagnosis is confirmed, immediate exploratory laparotomy should be proceeded. ${ }^{1,3,5,10}$ Endovascular repair is becoming an attractive treatment option.,4 


\section{CONFLICT OF INTEREST}

No potential conflict of interest relevant to this article was reported.

\section{ORCID}

Hyun Jin Bae (1) https://orcid.org/0000-0001-9167-6628 Byung-Wook Kim (1) https://orcid.org/0000-0002-2290-4954 Joon Sung Kim (1) https://orcid.org/0000-0001-9158-1012

\section{REFERENCES}

1. Šumskienė J, Šveikauskaitė E, Kondrackienė J, Kupčinskas L. Aorto-duodenal fistula: a rare but serious complication of gastrointestinal hemorrhage. A case report. Acta Med Litu 2016; 23:165-168.

2. Ranasinghe W, Loa J, Allaf N, Lewis K, Sebastian MG. Primary aortoenteric fistulae: the challenges in diagnosis and review of treatment. Ann Vasc Surg 2011;25:386.e1-e5.
3. Saers SJ, Scheltinga MR. Primary aortoenteric fistula. Br J Surg 2005;92:143-152.

4. Rhéaume P, Labbé R, Thibault E, Gagné JP. A rational, structured approach to primary aortoenteric fistula. Can J Surg 2008; 51:E125-E126.

5. Delgado J, Jotkowitz AB, Delgado B, Makarov V, Mizrahi S, Szendro G. Primary aortoduodenal fistula: pitfalls and success in the endoscopic diagnosis. Eur J Intern Med 2005;16:363-365.

6. Morrow C, Safi H, Beall AC Jr. Primary aortoduodenal fistula caused by Salmonella aortitis. J Vasc Surg 1987;6:415-418.

7. Lemos DW, Raffetto JD, Moore TC, Menzoian JO. Primary aortoduodenal fistula: a case report and review of the literature. J Vasc Surg 2003;37:686-689.

8. Song EM, Shim KN, Kang HW, et al. A case of primary aortoenteric fistula mimicking duodenal subepithelial tumor. Korean J Helicobacter Up Gastrointest Res 2012;12:50-53.

9. Ihama Y, Miyazaki T, Fuke C, et al. An autopsy case of a primary aortoenteric fistula: a pitfall of the endoscopic diagnosis. World J Gastroenterol 2008;14:4701-4704.

10. Bala M, Sosna J, Appelbaum L, Israeli E, Rivkind AI. Enigma of primary aortoduodenal fistula. World J Gastroenterol 2009;15: 3191-3193. 